\title{
MOLECULAR SPECTROSCOPY WITH ISO
}

\author{
Molecules in the Interstellar and Circumstellar Medium
}

J. CERNICHARO

CSIC, IEM, Dpto. Física Molecular, Serrano 123, E-28006 Madrid, Spain

\section{Introduction}

In spite of the important astrophysical output that could be obtained from infrared observations, the wavelength range $2-200 \mu \mathrm{m}$ has remained poorly studied due to the absorption of radiation produced by the Earth's atmosphere. The spectrometers on board the Infrared Space Observatory (ISO) provide a unique opportunity to study important molecular species through transitions which are inaccessible from the ground or airborne platforms. The ISO spectrometers offer the possibility to detect, with a reasonably good spectral resolution, the ro-vibrational transitions of important molecules. The SWS spectrometer cover the region $2-45 \mu \mathrm{m}$ and it is well adapted to the study of ro-vibrational transitions (stretching and bending modes) while the LWS spectrometer provides a powerful tool to observed the pure rotational transitions of light species like $\mathrm{H}_{2} \mathrm{O}$. In this review we present the main results on gas phase molecules obtained with the ISO-SWS and ISO-LWS spectrometers and also the low resolution CVF-ISOCAM spectrometer.

\section{Molecular Hydrogen}

Molecular hydrogen, $\mathrm{H}_{2}$, is the most abundant molecule in the interstellar and circumstellar medium. Due to its lack of permanent dipole moment this molecule can be only observed in the near and medium infrared through its ro-vibrational and quadrupolar rotational transitions. The abundance of $\mathrm{H}_{2}$ in molecular clouds has been established through comparisons of the emission of other molecules, like $\mathrm{CO}$, and the visual absorption using the relation $\mathrm{N}\left(\mathrm{H}_{2}\right) / \mathrm{Av}$ derived in the diffuse interstellar medium. The ISO-SWS spectrometer allows to observe the quadrupolar lines of $\mathrm{H}_{2}$ in the molecular clouds of the Milky Way and in external galaxies, to estimate directly the mass of $\mathrm{H}_{2}$, and to compare with the mass derived from $\mathrm{CO}$ data. In young stellar objects, like LKH $\alpha 224$, $\mathrm{LKH} \alpha 225$, Wesselius et al. (1996) have observed the $\mathrm{S}(1)$ up to $\mathrm{S}(7)$ lines of $\mathrm{H}_{2}$. The rotational levels of $\mathrm{H}_{2}$ seem to be populated with a thermal distribution at $500 \mathrm{~K}$ for LKH $\alpha 224$ and $800 \mathrm{~K}$ for $\mathrm{LKH} \alpha 225$. The amount of warm molecular hydrogen detected in these objects is 0.01 and 0.04 $\mathrm{M}_{\odot}$ respectively. The $\mathrm{S}(1)$ up to $\mathrm{S}(9)$ lines of $\mathrm{H}_{2}$ have been observed by Timmermann et al (1996) in the warm PDR of S140. The line intensity ratios are characteristic of fluorescent emission.

Valentijn et al. (1996) have observed the $\mathbf{S}(0), \mathrm{S}(1)$ and $\mathrm{S}(2)$ lines of $\mathbf{H}_{2}$ in the galaxy NGC6946. They have derived a mass of $510^{6} \mathrm{M}_{\odot}$ and a temperature of $170 \mathrm{~K}$ for the emitting gas. The observed warm $\mathrm{H}_{2}$ accounts for only $5-10 \%$ of the total molecular gas in the emitting region. Several $\mathrm{H}_{2}$ rotational lines have also detected in Arp220 by Sturm et al. (1996). They also conclude that the warm gas contains only $10 \%$ of the total molecular gas in Arp220. Similar results have been obtained by Kunze et al. (1996) in NGC4038/39 and by Rigopoulou et al. (1996) in the starburst galaxy NGC3256.

Finally, molecular hydrogen emission has been detected in the Helix Nebula by Cox et al. (in preparation) using the CVF-ISOCAM spectrometer. All the emission in the 5-17 $\mu \mathrm{m}$ band is dominated by the $\mathrm{H}_{2}$ lines. There is not trace of emission from PAHs characteristic of less evolved $\mathrm{PN}$ as NGC7027. 


\section{Water Vapour in the Interstellar Medium}

The determination of the abundance of water vapour is a long standing problem in modern astrophysics and despite many ground-based and airborne observations, this issue is still open. Water was discovered by Cheung et al. (1960) through the maser emission of its $6_{16}-5_{23}$ rotational transition at $22 \mathrm{GHz}$. Many other maser transitions have since then been observed towards various sources including stars, star-forming regions and extragalactic objects: most notably in the submillimeter range (Cernicharo et al. 1990; Menten \& Melnick 1991 and references therein). Cornicharo et al. (1994) reported widespread water vapour emission in Orion in the $3_{13}-2_{20}$ rotational transition at $183.3 \mathrm{GHz}$ which was shown to be dominated by a weak, and spatially extended, maser emission. From these observations a water abundance $\simeq 10^{-5}$ was derived. However, these observations require exceptionally good weather conditions. The spectrometers on board the Infrared Space Observatory (ISO) are very well adapted to the study of the emission/absorption of water vapour transitions. Water emission has been detected towards young stellar objects by Liscau et al. (1996) and Saraceno et al. (1996). The physical conditions derived from the observed water vapour lines and the high excitation lines of $\mathrm{CO}$ in these objects correspond to shocked gas with $\mathrm{T}_{K} \simeq 300 \mathrm{~K}$ and $x\left(\mathrm{H}_{2} \mathrm{O}\right) \simeq 10^{-5}$. Water vapour is probably produced in these shocked regions through evaporation of water ice from dust grain mantles. The SWS spectrometer has been used by van Dishoeck and collaborators to study the ro-vibrational transitions of the bending mode of $\mathrm{H}_{2} \mathrm{O}$ in the direction of the bright infrared sources associated with AFLG2591, NGC7538, AFLG2136 and AFGL4176 (van Dishoeck and Helmich 1996., Helmich et al. 1996). The rotational temperature they derived is $300 \mathrm{~K}$ and the water vapour abundance is similar to that derived by Cernicharo et al (1994) in the direction of ORion-IRc2, i.e, $\simeq 10^{-5}$. Finally, Cernicharo et al (1997a) have observed the pure rotational transitions of $\mathrm{H}_{2} \mathrm{O}$ and $\mathrm{H}_{2}{ }^{18} \mathrm{O}$ with the grating-LWS and the FP-LWS in the direction of SgrB2. They show that water is widespread and detected in absorption with $x\left(\mathrm{H}_{2} \mathrm{O}\right) \simeq 10^{-5}$. They conclude that water vapour excitation in objects that are optically thick at $100-200 \mu \mathrm{m}$ is dominated by dust emission.

\section{Evolved Stars}

About 30 pure rotational lines of water vapour have been detected in the oxygen-rich AGB star W Hya by Barlow et al. (1996). The water vapour abundance derived from the modelling of the data is $10^{-4}$. Neufeld et al. (1996) have observed 4 high excitation transitions of $\mathrm{H}_{2} \mathrm{O}$ with the SWSFP spectrometer in the same object. The ro-vibrational transitions of the bending mode of $\mathrm{H}_{2} \mathrm{O}$, together with the stretching mode of $\mathrm{OH}$ between 3-3.5 $\mu \mathrm{m}$ and the stretching mode of $\mathrm{CO}_{2}$ at 4.2 $\mu \mathrm{m}$, have been observed for the first time in the evolved star NML Cyg by Justtanont et al. (1996) using the SWS spectrometer. Many evolved stars have observed with the LWS spectrometer in the GT proposal of Mike Barlow. The far-infrared spectrum of IRC+10216 constitutes a prototype of the molecular emission in carbon-rich stars in the AGB phase. Cernicharo et al. (1996b) have analyzed the LWS data on this object and conclude that the HCN rotational emission in its ground and vibrationally excited states contributes to the cooling of the gas in a similar way than $\mathrm{CO}$. They have derived an abundance for $\mathrm{HCN}$ of $310^{-5}$. The pure rotational lines of the bending modes of HCN are particularly strong and most of that emission arises from the inner regions of the envelope. In CRL2688, a C-rich object evolving towards the PN stage, Cox et al (1996) have modelled the $\mathrm{CO}$ emission as arising from a shocked region in the inner part of the envelope. This shocked gas is also responsible for the emission of $\mathrm{H}_{2}$ vibrationally excited. Finally, Liu et al. (1996) have observed the PN NGC7027, showing that the emission is dominated by atomic lines and the CO rotational lines. In this object Cernicharo et al (1997b) have discovered the pure rotational lines of $\mathrm{CH}^{+}$(see below).

\section{5. $\mathrm{CH}^{+}$}

$\mathrm{CH}^{+}$was one of the first molecules detected in Astrophysics through its ${ }^{1} \Pi{ }^{1} \Sigma$ electronic transition at optical wavelengths. This molecule is abundant in the diffuse interstellar medium and during many years the chemical reactions involved in its formation have been subject of inportant controversy. Cernicharo et al (1997b) have reported the discovery of several pure rotational transitions of $\mathrm{CH}^{+}$in the LWS spectrum of NGC 7027. The strong line at $179.62 \mu \mathrm{m}$ and the lines at 119.90 
and 90.03 $\mu \mathrm{m}$ (reported as unidentified by Liu et al. 1996), whose frequencies are in harmonic relation $2: 3: 4$, are shown to arise from the $\mathrm{J}=2-1,3-2$, and 4-3 rotational transitions of $\mathrm{CH}^{+}$. This identification is strengthened by the detection in the LWS spectra of the next two rotational lines of $\mathrm{CH}^{+}$, at 72.17 and $60.22 \mu \mathrm{m}$. Cernicharo et al $(1997 \mathrm{~b})$ have derived a rotational temperature for the $\mathrm{CH}^{+}$lines of $160 \mathrm{~K}$, a volume density of a few $10^{7} \mathrm{~cm}^{-3}$, and a $\mathrm{CH}^{+} / \mathrm{CO}$ abundance ratio of $0.2-110^{-3}$. Given the carbon-rich nature of NGC 7027 and the strong UV radiation field in its inner regions it is thus not too surprising to find $\mathrm{CH}^{+}$in this prototypical PN. However, this is the first time that $\mathrm{CH}^{+}$has been seen through its pure rotational spectrum. Recently, the transitions $\mathrm{J}=9-8$ and $\mathrm{J}=10-9$ have also been detected in NGC 7027 (Cernicharo et al, in preparation) with the SWS spectrometer $(\Delta \lambda / \lambda \simeq 1600)$. Taking into account the dipole moment of $\mathrm{CH}^{+}(1.7 \mathrm{D})$ and the structure of its rotational levels (the $J=10$ level is at $\simeq 2200 \mathrm{~K}$ ), the rotational transitions of this molecular species constitute a unique tool to trace the physical conditions of the PDRs of the interstellar and circumstellar medium.

\section{Other molecules}

The sensitivity of the ISO spectrometers allows to search for new molecular species. Together with $\mathrm{CH}^{+}$, other light species, like $\mathrm{CH}_{2}, \mathrm{NH}, \mathrm{SH}, \mathrm{SiH}, \mathrm{FH}$ could be detected in the far-infrared. However, these molecules are not very abundant and, in order to have unambiguous frequency assignments, the Fabry-Perot spectrometers must be used. Recently, Neufeld et al. (1997) have reported the detection of the $\mathrm{J}=2-1$ line of $\mathrm{FH}$ in the direction of $\mathrm{SgrB} 2$. The very large infrared flux in that direction allows to search for weak features. The abundance that they have derived for $\mathrm{FH}$ is $10^{-9}$. Another Fluorine-bearing molecule, AlF, has been previously reported by Cernicharo \& Guélin (1987) in the direction of the circumstellar envelope of IRC+10216, but it is the first time that a Fluorine bearing molecule has been detected in the interstellar medium.

Triatomic carbon has been detected by Cernicharo et al (1996a, and in preparation) in IRC+10216 through its ro-vibrational transitions in the far-infrared. The molecule was already detected in evolved stars through its stretching mode at $5 \mu \mathrm{m}$, but the new observation of the low-lying bending mode at $62 \mathrm{~cm}^{-1}$ allow to search for this species in the interstellar medium. Recently, Cernicharo et al (in preparation) have detected two lines of $\mathrm{C}_{3}$ in the direction of $\mathrm{SgrB} 2$. This observation constitutes the first detection of this molecule in the interstellar medium. Another important organic molecule, $\mathrm{CH}_{4}$, has been detected by Van Dishoeck and coworkers. Most of the LWS and SWS observations of the interstellar and circumstellar medium are still under analysis. In the next months we could expect the detection of new molecular species. In particular, the guarantee time frequency sweeps of SgrB2 and Orion-Irc2 will probably provide an excellent data base for the study of gas-phase chemistry.

\section{References}

Barlow M.J., Nguyen-Rieu, Truong-Bach et al:1996, A.\&A., 315, L241

Cernicharo J., Thum C., Hein H., Garcia P., \& Mattiocco F. 1990, AA 231, L15.

Cernicharo J., González-Alfonso E., Alcolea J., Bachiller R., \& John D. 1994, ApJ, 432, L59

Cernicharo J., Bachiller R., \& González-Alfonso E. 1996a, AA, 305, L5.

Cernicharo J., Barlow M., González-Alfonso E., 1996b, A.\&A., 315, L201

Cernicharo J., Lim T., Cox P., et al. 1997a, A\&A, 323, L25

Cernicharo J.. X-Liu, González-Alfonso E., et al. 1997b, ApJ, 483, L65

Cox P., González-Alfonso E., Barlow M., et al., 1996, A.\&A., 315, L265

Cheung A.C., Rank D.M., Townes C.H., Thornton D.D., \& Welch W.J. 1969, Nature, 221, 626

Helmicl $_{1}$ F.P., van Dishoeck E.F., Black J.H. et al, A.\&A. 315, L173

Justtanont K., deJong T., Helmich F.P., et al., 1996, A.\&A., 315, L217

Kunze D., Rigopoulou D., Lutz D., et al., 1996, A.\&A., 315, L101

Liseau R., Ceccarelli C., Larsson B., et al., 1996, A.\&A., 315, L181

Liu X.-W., Barlow M.J., Nguyen-Q-Rieu, et al., 1996, A.\&A., 315, L257

Menten, K., \& Melnick, G.J. 1991, ApJ, 377, 647

Neufeld D.A., Chen W., Melnick G.J. et al., 1996, A.\&A., 315, 237

Rigopoulou D., Lutz D., Genzel R., et al., 1996, A.\&A., 315, L125

Saraceno P., Ceccarelli C., Clegg P., et al., 1996, A.\&A., 315, L293

Sturm E., Lutz D., Genzel R., 1996, A.\&A., 315, L 133

Timmermann R., Bertoldi F., Wright C.M., et al., 1996, A.\&A., 315, L281

Valentijn E.A., van der Werf P.P, de Graauw T, et al., 1996, A.\&A., 315, L145

van Dishoeck E., Helmich .:1996, A.\&A., 315, L177

Wesselius P.R., van der Ancker M.E., Young E.T., et al., 1996, A.\&A., 315, L197 